Article

\title{
Frequency-Splitting-Free Synchronous Tuning of Close-Coupling Self-Oscillating Wireless Power Transfer
}

\author{
Po Hu*, Jieshuai Ren and Wenan Li \\ School of Electrical Engineering, Wuhan University, 299 Bayi Road, Wuchang, Wuhan 430072, China; \\ 2011202070070@whu.edu.cn (J.R.); wenanli@whu.edu.cn (W.L.) \\ * Correspondence: phu@whu.edu.cn; Tel.: +86-153-4221-2426; Fax: +86-27-6252-7627
}

Academic Editor: Hongjian Sun

Received: 1 May 2016; Accepted: 20 June 2016; Published: 27 June 2016

\begin{abstract}
The synchronous tuning of the self-oscillating wireless power transfer (WPT) in a close-coupling condition is studied in this paper. The Hamel locus is applied to predict the self-oscillating points in the WPT system. In order to make the system operate stably at the most efficient point, which is the middle resonant point when there are middle resonant and split frequency points caused by frequency-splitting, the receiver (RX) rather than the transmitter (TX) current is chosen as the self-oscillating feedback variable. The automatic delay compensation is put forward to eliminate the influence of the intrinsic delay on frequency tuning for changeable parameters. In addition, the automatic circuit parameter tuning based on the phase difference is proposed to realize the synchronous tuning of frequency and circuit parameters. The experiments verified that the synchronous tuning proposed in this paper is effective, fully automatic, and more robust than the previous self-oscillating WPT system which use the TX current as the feedback variable.
\end{abstract}

Keywords: wireless power transfer (WPT); close coupling; self-oscillation; Hamel locus; frequency-splitting; synchronous tuning

\section{Introduction}

Recently, the magnetic coupling resonant WPT has found widespread applications, such as wireless sensors, electrical vehicles, and wireless household appliances [1-4]. This type of WPT system is non-radiative with ignorable radiative loss [5], and often operates in the close-coupling condition where the transfer range is approximately equal to the diameter of the coils. Therefore, the transfer efficiency is not only high, but also remains nearly unchanged in a certain range even when the gap and misalignment varies $[1,3,6]$. In order to satisfy the condition of close-coupling, the operating frequency is often set to several $\mathrm{kHz}$-several $\mathrm{MHz}$, and the quality-factor $(Q)$ should be as high as 200-2000 [3,5,7,8]. However, many factors, e.g., environmental changes, aging of the resonant tanks, and errors of individual components of mass production, may lead to variations of the operating frequency or circuit parameters including the inductances and the capacitances, which result in severe detuning in the high- $Q$ resonant tanks [8-10]. To solve these problems, the automatic synchronous tuning method considering the frequency tuning and circuit parameter tuning, i.e., the tuning of the circuit parameters such as capacitances and inductances, should be investigated. The former makes the inverter operate in zero-current switching, and the latter ensures the resonant frequency of the transmitter (TX) and receiver (RX) is the same.

Much of the previous research in this area of WPT focuses on the one-off tuning methods rather than the fully-automatic tuning which keeps the system resonant all the time, shown in Figure 1a,b, respectively. For the frequency tuning, the methods based on frequency-scanning are often used $[1,2,6]$. 
The tuning stops when the resonant frequency is found, so the detuning detection and restart control are needed when the tuning stops. In addition, power is not normally transferred in the tuning process. Many of the circuit parameter tuning methods are also one-off tuning or dynamically-stable tuning, such as the disturbance observation method and the intelligent circuit parameter searching method coming from the automatic impedance matching $[6,8,11,12]$.

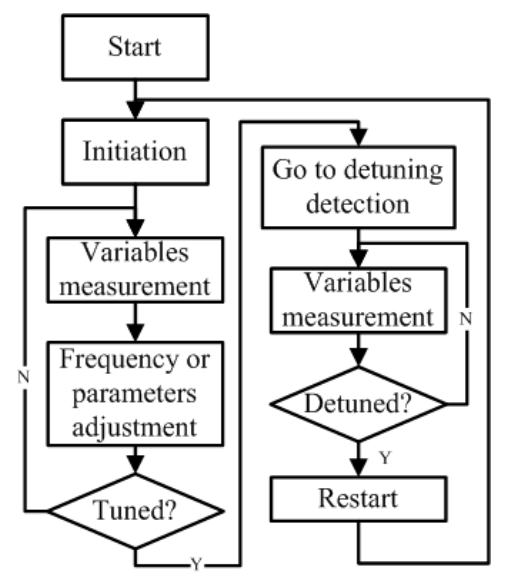

(a)

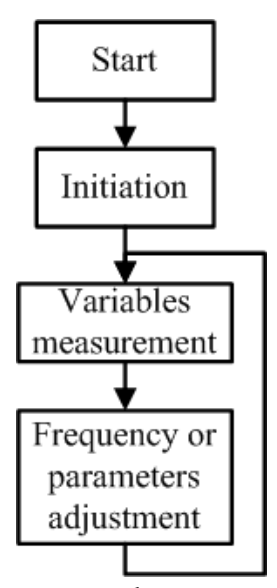

(b)

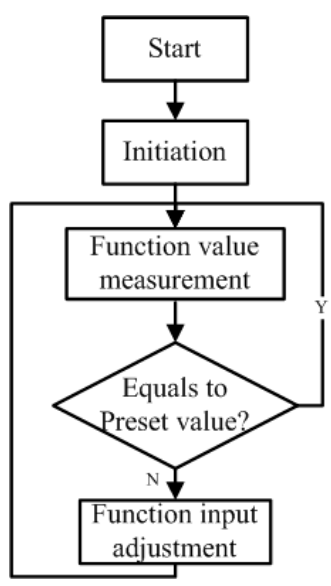

(c)

Figure 1. Flowcharts of tuning methods: (a) one-off tuning with restart when detuned; (b) fully-automatic tuning; and (c) tuning according to functions.

The fully automatic frequency or circuit parameter tuning is also studied before. The frequency control by detecting the detuning loop current in inverter $[9,13]$ is complex, and cannot be used in high-power applications for long. The circuit parameters setting, based on the function between the measurement and the control variable, is precise and easily conducted, as shown in Figure 1c, but some of these methods are based on table or online circuit parameter calculations $[6,14,15]$, and the precise measurement of the circuit parameter values, which must be obtained in advance, are uncertain or changeable in applications. In addition, the previous circuit parameter tuning is not always effective for the variable frequency caused by automatic frequency tuning.

The self-oscillating control method makes the inverter flip at the moment when the TX current crosses zero, so the operating frequency of the inverter, i.e., the self-oscillating frequency, equals to the resonant frequency even if circuit parameters change $[13,16,17]$, which is suitable for automatic frequency tuning. However, due to the frequency-splitting in close coupling condition, there may be multiple resonant points, such as the middle resonant point, odd, and even splitting points. The previous self-oscillating methods make the system operate at one of the splitting points, but not always with maximum efficiency [18-20], and some studies indicate that the self-oscillating system becomes unstable under disturbance because it will hop from one splitting point to another when the circuit parameters change [17].

Furthermore, the self-oscillating control cannot always keep the system resonant since the intrinsic delay in the components of the feedback loop can be added up to several hundred ns and makes the TX current lag behind the output voltage of the inverter, not only detuning the system, but also doing harm to the voltage source inverter, especially at high frequency $[9,17,21,22]$. Though the constant delay could be compensated by the negative hysteresis link [21] and linear network [22], these methods are only useful for the fixed frequency operation rather than the variable frequency operation caused by frequency tuning. The method in [23] achieves self-adaptive delay compensation, but it is only verified in loose coupling and needs deeper research.

To solve the problems mentioned above, in this paper, the self-oscillating frequency and its variation trend with circuit parameter changes are analyzed with different feedback variables. The WPT system is modeled as a non-linear switched system, and the Hamel locus is applied since it is precise 
and intuitive in the prediction of self-oscillating frequency and its variation trend, and is easy in modelling the delay link $[21,24]$. The series-series (SS) topology WPT system is chosen as an example because the resonant frequency in the SS topology is independent of the load and the coupling coefficient $[12,18]$. The TX current and RX current are treated as the self-oscillating feedback variables to analyze the self-oscillating characteristics, and the RX current is chosen for feedback because the system operates steadily in the middle resonant frequency with the maximum efficiency, regardless of the frequency splitting. On the basis of analysis of the variation trend between the self-oscillating frequency and the delay in the feedback loop, a novel automatic frequency tuning with automatic delay compensation is proposed, in which only frequency measurement and delay setting are needed. Furthermore, to realize the automatic frequency tuning, a function of the detuning and the phase difference between the currents in TX and RX is also investigated, and the circuit parameter tuning with the phase difference detection and capacitance adjustment is proposed to realize the synchronous tuning. The experiments verify the effectiveness and robustness of the synchronous tuning method.

\section{Analysis of the Self-Oscillating Feedback Methods}

The self-oscillating frequency characteristics, using the TX current $i_{1}$ or the RX current $i_{2}$ as the feedback variable, are analyzed respectively. The power circuit and its linear equivalent circuit of the WPT system are shown in Figure 2a,b, respectively, and the block diagram for control is shown in Figure 3.

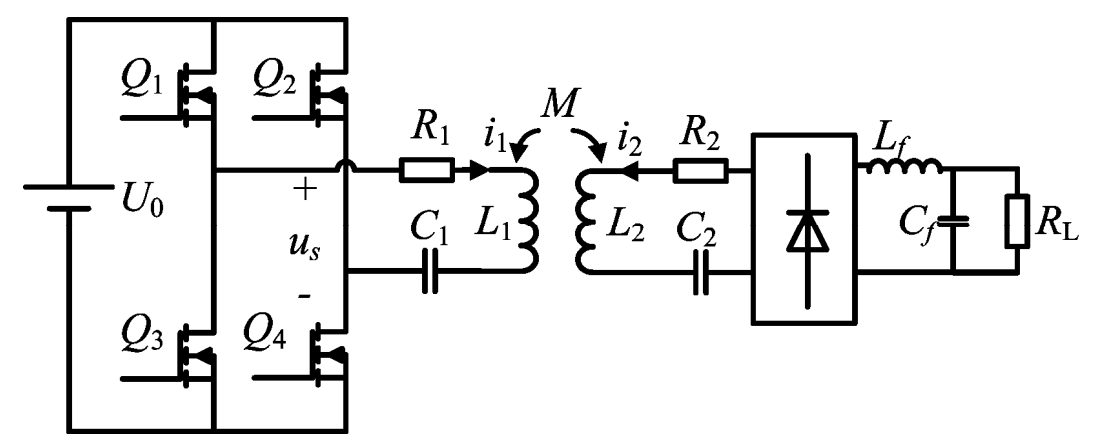

(a)

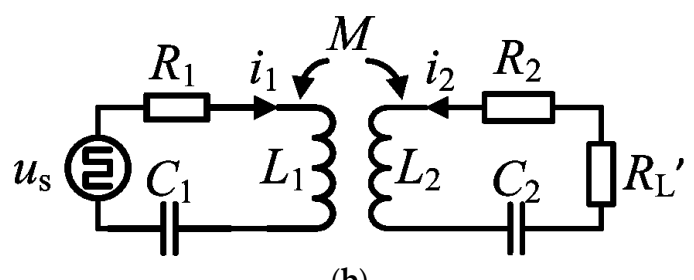

(b)

Figure 2. Power circuit and linear equivalent circuit of wireless power transfer (WPT) system: (a) power circuit; and (b) linear equivalent circuit.

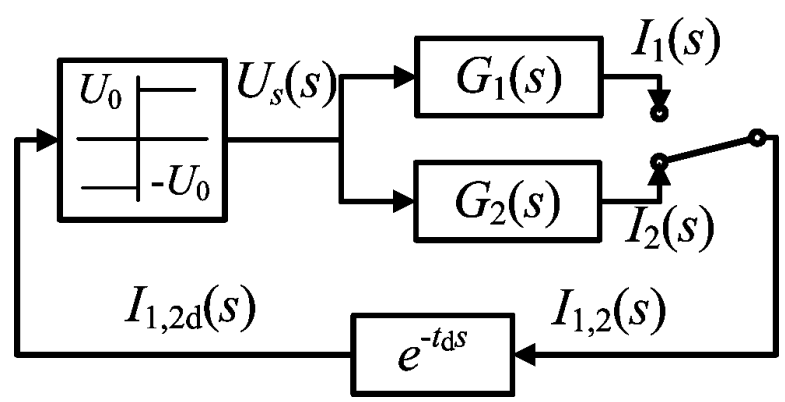

Figure 3. Block diagram of control system. 
The power circuit of the WPT system consists of the DC power source, inverter, resonant tanks, rectifier, filter, and load. The DC power source $U_{0}$ and the high-frequency inverter composed of MOSFETs $Q_{1}-Q_{4}$ are modeled as a square wave source in Figure $2 b$. The resonant tanks contains the coils with the inductances $L_{1}, L_{2}$, the capacitors with the capacitances $C_{1}, C_{2}$, and the two equivalent resistors, whose resistance $R_{1}$ in the TX, is the sum of resistances of the source, inverter, and TX coil, and the resistance $R_{2}$ in the RX is the sum of resistances of the RX coil, rectifier, and filter. The rectifier, filter $L_{f}, C_{f}$, and load $R_{\mathrm{L}}$ can be equivalent to $R_{\mathrm{L}}{ }^{\prime}$ in Figure $2 \mathrm{~b}$, where $R_{\mathrm{L}}{ }^{\prime}=\left(8 / \pi^{2}\right) R_{\mathrm{L}}[12,25] . M$ is the mutual inductance, $M=k \sqrt{L_{1} L_{2}}$, and $k$ is the coefficient of mutual inductance.

In Figure 3, the inverter is modeled as an ideal relay link because the dead time in SiC MOSFETs is only several ns and, therefore, ignorable. In order to analyze the frequency characteristics with the feedback variables $i_{1}$ or $i_{2}$, the Hamel locus functions $H_{1}(f)$ and $H_{2}(f)$ without the delay $e^{-t_{d} s}$ are derived.

The transfer functions $G_{1}(s)$ and $G_{2}(s)$ can be obtained by solving the complex frequency domain Equation (1) describing the linear part. For the convenience, the circuit parameters of the system is set to symmetrical [25], i.e., $R=R_{1}=R_{2}+R_{\mathrm{L}^{\prime}}{ }^{\prime} L=L_{1}=L_{2}, C=C_{1}=C_{2}$ in (2).

$$
\begin{gathered}
\left\{\begin{array}{c}
I_{1}(s)\left(R_{1}+s L_{1}+\frac{1}{s C_{1}}\right)-I_{2}(s) s M=U_{s}(s) \\
-I_{1}(s) s M+I_{2}(s)\left(R_{2}+R_{L}+s L_{2}+\frac{1}{s C_{2}}\right)=0
\end{array}\right. \\
\left\{\begin{array}{l}
G_{1}(s)=\frac{I_{1}(s)}{U_{s}(s)}=\frac{L C^{2} s^{3}+R C^{2} s^{2}+C s}{s^{4}\left(C^{2} L^{2}-C^{2} M^{2}\right)+2 C^{2} L R s^{3}+s^{2}\left(C^{2} R^{2}+2 L C\right)+2 C R s+1} \\
G_{2}(s)=\frac{I_{2}(s)}{U_{s}(s)}=\frac{C^{2} M s^{3}}{s^{4}\left(C^{2} L^{2}-C^{2} M^{2}\right)+2 C^{2} L R s^{3}+s^{2}\left(C^{2} R^{2}+2 L C\right)+2 C R s+1}
\end{array}\right.
\end{gathered}
$$

The step response of Equation (2) is:

$$
q_{1,2}(t)=a_{1} \sin \left(\omega_{1} t\right) e^{-b_{1} t} \pm a_{2} \sin \left(\omega_{2} t\right) e^{-b_{2} t}
$$

where the sign " \pm " is " + " in $q_{1}(t)$ and " - " in $q_{2}(t)$, and:

$$
\left\{\begin{array}{lll}
a_{1}=\frac{1}{\left(-R^{2}+4(L-M) / C\right)^{1 / 2}} & b_{1}=\frac{R}{2(L-M)} & \omega_{1}=\frac{\left(-R^{2}+4(L-M) / C\right)^{1 / 2}}{2(L-M)} \\
a_{2}=\frac{1}{\left(-R^{2}+4(L+M) / C\right)^{1 / 2}} & b_{2}=\frac{R}{2(L+M)} & \omega_{2}=\frac{\left(-R^{2}+4(L+M) / C\right)^{1 / 2}}{2(L+M)}
\end{array}\right.
$$

It can be seen from Equation (4) that the step responses of $i_{1}$ and $i_{2}$ are the superposition of two exponentially-decayed sinusoidal functions.

The square wave responses $i_{1}(t)$ and $i_{2}(t)$ are the superposition of a series of positive and negative step responses $q_{1}(t)$ and $q_{2}(t)$, as shown in (5), and the derivatives $\mathrm{d} i_{1}(t) / \mathrm{d} t$ and $\mathrm{d} i_{2}(t) / \mathrm{d} t$ are given in (6):

$$
\begin{aligned}
i_{1,2}(t) & =2 U_{0}\left(\sum_{n=1}^{\infty} q_{1,2}(t+n T)-\sum_{n=2}^{\infty} q_{1,2}\left(t-\frac{T}{2}+n T\right)\right) \\
& =a_{1} U_{0} \frac{e^{-b_{1} t} \sin \omega_{1}\left(t-\frac{1}{2} T\right)+e^{-b_{1}\left(t-\frac{1}{2} T\right)} \sin \omega_{1} t}{\cosh \frac{b_{1} T}{2}+\cos \frac{\omega_{1} T}{2}} \pm a_{2} U_{0} \frac{e^{-b_{2} t} \sin \omega_{2}\left(t-\frac{1}{2} T\right)+e^{-b_{2}\left(t-\frac{1}{2} T\right)} \sin \omega_{2} t}{\cosh \frac{b_{2} T}{2}+\cos \frac{\omega_{2} T}{2}} \quad t \in\left(0, \frac{T}{2}\right] \\
\frac{d i_{1,2}(t)}{d t} & =a_{1} U_{0} \frac{\left(-b_{1} e^{-b_{1} t} \sin \omega_{1}\left(t-\frac{1}{2} T\right)+\omega_{1} e^{-b_{1} t} \cos \omega_{1}\left(t-\frac{1}{2} T\right)-b_{1} e^{-b_{1}\left(t-\frac{1}{2} T\right)} \sin \omega_{1} t+\omega_{1} e^{-b_{1}\left(t-\frac{1}{2} T\right)} \cos \omega_{1} t\right)}{\cosh \frac{b_{1} T}{2}+\cos \frac{\omega_{1} T}{2}} \quad t \in\left(0, \frac{T}{2}\right] \\
& \pm a_{2} U_{0} \frac{\left(-b_{1} e^{-b_{2} t} \sin \omega_{2}\left(t-\frac{1}{2} T\right)+\omega_{2} e^{-b_{2} t} \cos \omega_{2}\left(t-\frac{1}{2} T\right)-b_{2} e^{-b_{2}\left(t-\frac{1}{2} T\right)} \sin \omega_{2} t+\omega_{2} e^{b_{2}\left(t-\frac{1}{2} T\right)} \cos \omega_{2} t\right)}{\cosh \frac{b_{2} T}{2}+\cos \frac{\omega_{2} T}{2}}
\end{aligned}
$$


Substituting the period fixed point $t=T / 2=1 / 2 f$ into (5) and (6) gives the frequency characteristics of $i_{1}, i_{2}$, i.e., the Hamel locus functions, and their derivatives:

$$
\begin{gathered}
H_{1,2}(f)=i_{1,2}\left(\frac{1}{2 f}\right)=a_{1} U_{0} \frac{\sin \frac{\omega_{1}}{2 f}}{\cosh \frac{b_{1}}{2 f}+\cos \frac{\omega_{1}}{2 f}} \pm a_{2} U_{0} \frac{\sin \frac{\omega_{2}}{2 f}}{\cosh \frac{b_{2}}{2 f}+\cos \frac{\omega_{2}}{2 f}} \\
H \prime_{1,2}(f)=\frac{d i_{1,2}\left(\frac{1}{2 f}\right)}{d t}=a_{1} U_{0} \frac{\omega_{1} e^{-\frac{b_{1}}{2 f}}-b_{1} \sin \frac{\omega_{1}}{2 f}+\omega_{1} \cos \frac{\omega_{1}}{2 f}}{\cosh \frac{b_{1}}{2 f}+\cos \frac{\omega_{1}}{2 f}} \pm a_{2} U_{0} \frac{\omega_{2} e^{-\frac{b_{2}}{2 f}}-b_{2} \sin \frac{\omega_{2}}{2 f}+\omega_{2} \cos \frac{\omega_{2}}{2 f}}{\cosh \frac{b_{2}}{2 f}+\cos \frac{\omega_{2}}{2 f}}
\end{gathered}
$$

The Hamel loci with the feedback variables $i_{1}$ and $i_{2}$ are shown in Figure $4 \mathrm{a}, \mathrm{b}$ respectively, where $U_{0}=20 \mathrm{~V}, R=3 \Omega, L=50 \mu \mathrm{H}, C=1750 \mathrm{pF}, k=0.056$, and the resonant frequency $f_{\text {res }}=538.04$ $\mathrm{kHz}$. The system under the chosen frequency is suitable for full bridge inverters, and the close coupling condition $k^{2} Q^{2}=17.857>1$ is satisfied $[20,25]$. The frequency range in the loci is $0.5 f_{\text {res }}-2 f$ res. The arrows in Figure 4 indicate the direction of the frequency increase. The intersections of the negative $x$-axis and the loci are self-oscillating points, and among them the ones crossing from the negative to positive are stable ones, while the others are unstable ones [21,24].

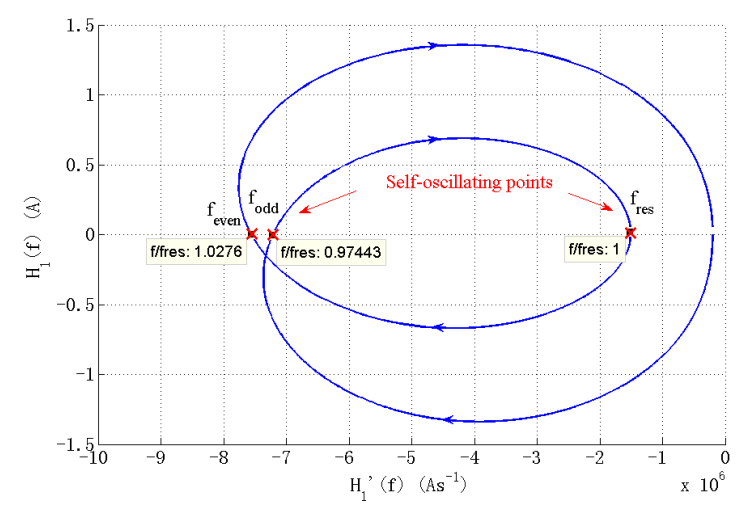

(a)

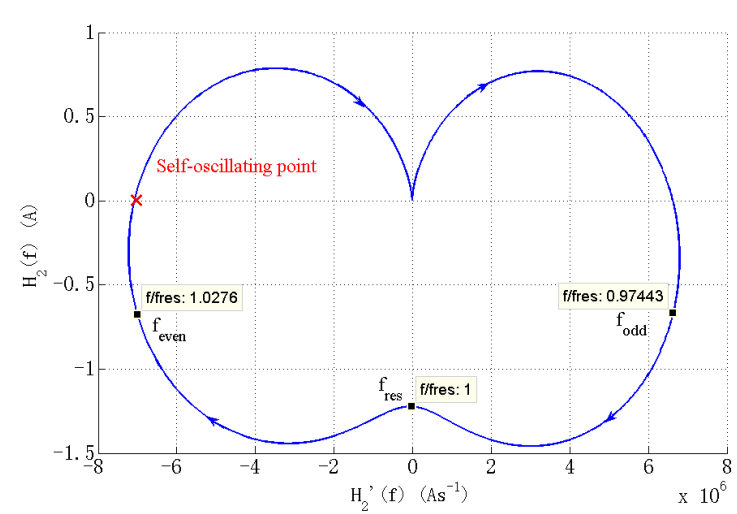

(b)

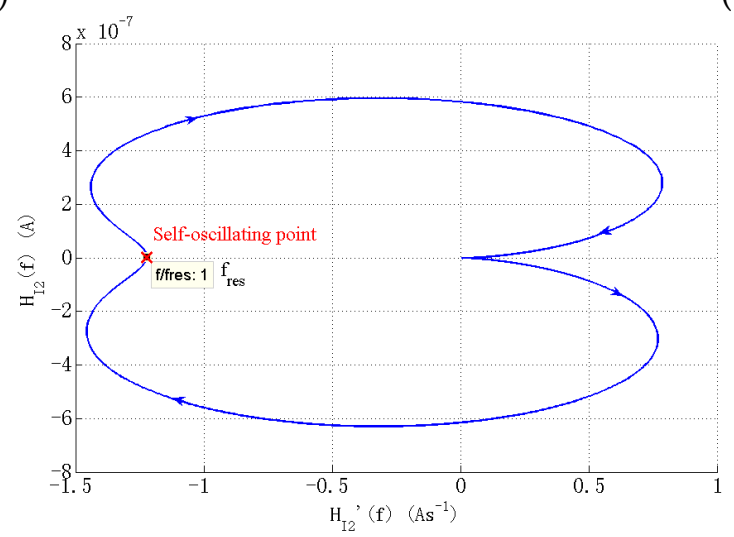

(c)

Figure 4. Hamel loci of different feedback methods: (a) feedback variable $i_{1} ;(\mathbf{b})$ feedback variable $i_{2}$; and (c) feedback variable $i_{2}$ with an integrator.

For the feedback variable $i_{1}$, the inverter flips when $i_{1}$ crosses zero, so the self-oscillating points are in coincidence with the middle resonant point or the splitting points, as shown in Figure 4a. Unfortunately, the middle resonant point with the frequency $f_{\text {res }}$ is unstable, and both of the odd and even splitting points [26] with the splitting frequencies $f_{\text {odd }}$ and $f_{\text {even }}$ are stable. Studies in [18-20] indicate that, in the close coupling 2-coil WPT systems, though the power at $f_{\text {res }}$ is smaller than the 
one at $f_{\text {odd }}$ or $f_{\text {even, }}$ the efficiency is higher at $f_{\text {res. }}$. The reason why the efficiency is more important than power is that the high power can be easily obtained by high voltage source.

For the feedback variable $i_{2}$, the inverter flips when $i_{2}$ crosses zero, so the self-oscillating point is not equal to the middle resonant point or the splitting points because of the phase difference between $i_{1}$ and $i_{2}$, as shown in Figure $4 \mathrm{~b}$. The middle resonant point, however, is located at the intersection between the loci and the negative $y$-axis, and can be shifted to the negative $x$-axis by adding an integrator in $G_{2}(s)$ to yield $90^{\circ}$ phase shift, as shown in Figure 4c.

The circuit parameters disturbance occurs when the inductances and the capacitances of the resonant tanks change in the varying environment. The self-oscillating characteristics under parameters disturbance are analyzed by plotting the Hamel loci of the system with asymmetric parameters. Without loss of generality, the variation in $C_{2}$ represents the detuning. The explicit Hamel locus functions are difficult to derive, so the Symbolic Math Toolbox of the MATLAB is used. Figure 5a,b shows the Hamel loci for the feedback variables $i_{1}$ and $i_{2}$, respectively, with the $C_{2}$ increased to $1900 \mathrm{pF}$. An integrator is added in Figure $5 \mathrm{~b}$ to produce a $90^{\circ}$ phase shift. In Figure $5 \mathrm{a}$, there is only one stable self-oscillating point rather than two, as in Figure $4 a$, and this point is in the vicinity of the even splitting point. Thus, if the system operates at the odd splitting point originally, this circuit parameter disturbance will make the system go into the even splitting point after being retuned, which means the robustness for the feedback variable $i_{1}$ is low. While in Figure $5 \mathrm{~b}$, there is only one stable self-oscillating point when detuning, so the system goes back to the middle resonant point after being retuned. As a result, $i_{2}$ is more suitable for feedback.

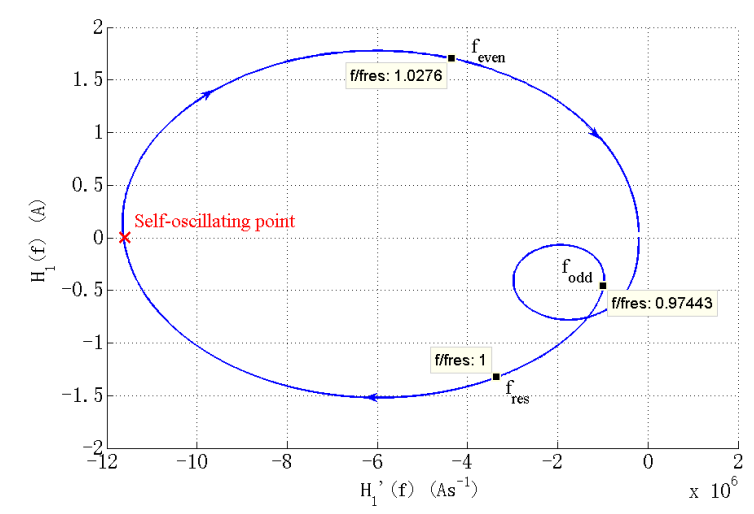

(a)

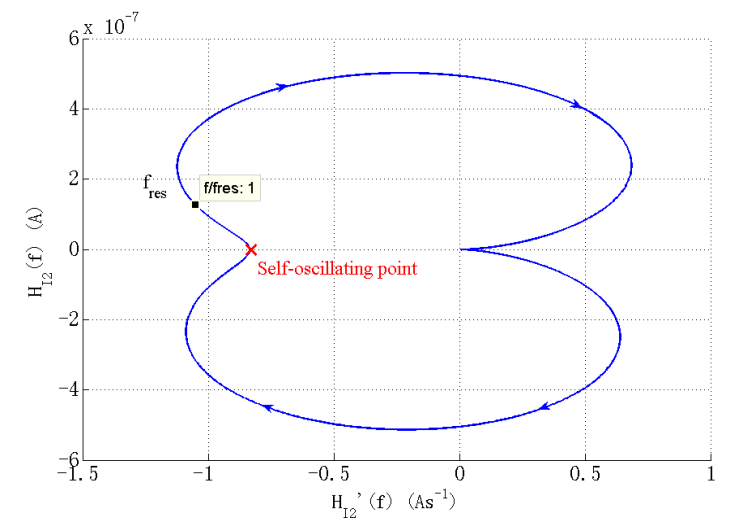

(b)

Figure 5. Hamel loci when $C_{2}$ increases: (a) feedback variable $i_{1}$; and (b) feedback variable $i_{2}$ with an integrator.

\section{Automatic Delay Compensation in Self-Oscillating WPT System}

The frequency characteristics and delay compensation of the self-oscillating WPT system with the delay $e^{-t_{d} s}$ are analyzed in this section. The transfer functions of $i_{1}$ and $i_{2}$ with the delay are:

$$
G_{d 1,2}(s)=G_{1,2}(s) e^{-t_{d} s}
$$

The Hamel locus function $H_{d 1,2}(f)$ with the delay are obtained by derivation similar to Equations (3)-(7). The implicit function (Equation (10)) of the delay $t_{d}$ and the self-oscillating period $T$ is obtained by letting $H_{d 1,2}(f)=0$, and the function is plotted in Figure 6 in the different range of $t_{d}$.

$$
F_{1,2}\left(t_{d}, T\right)=H_{d 1,2}(T / 2)=0
$$




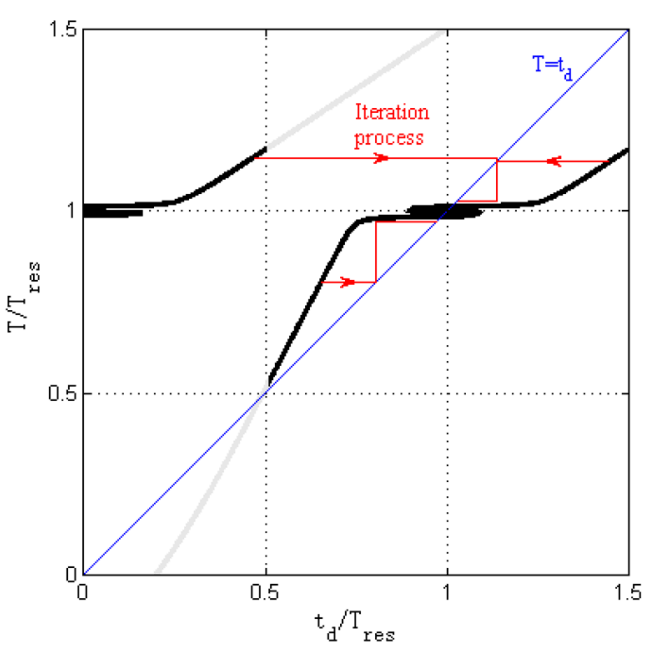

(a)

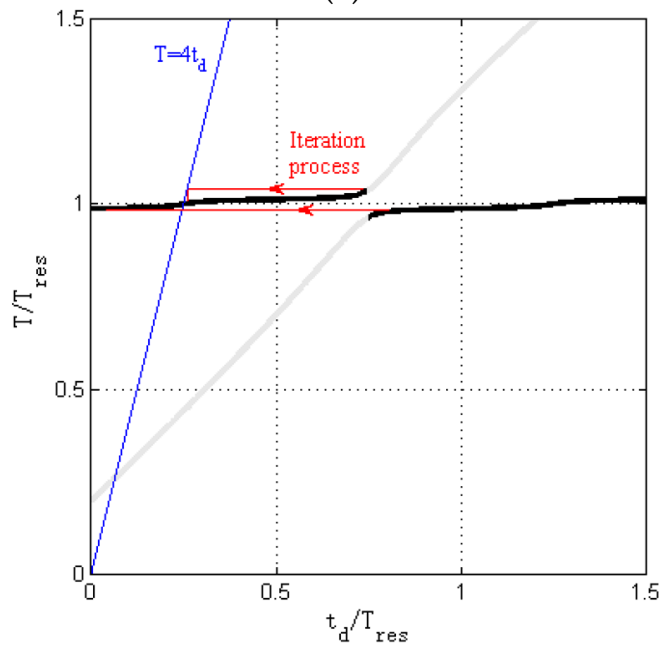

(c)

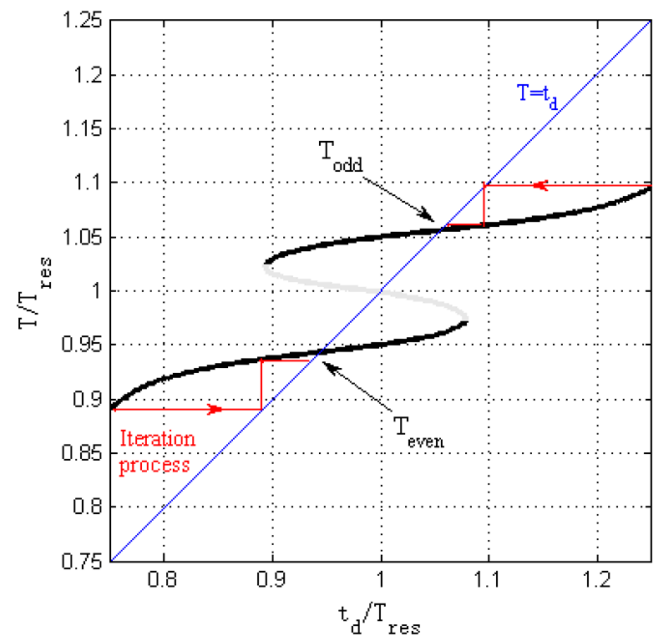

(b)

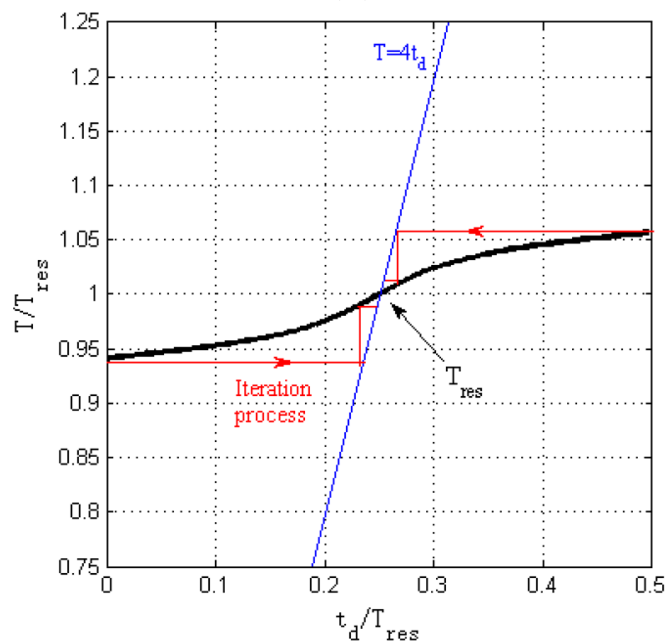

(d)

Figure 6. Function $F_{1,2}\left(t_{d}, T\right)$ : (a) $F_{1}\left(t_{d}, T\right)$ in full range; (b) $F_{1}\left(t_{d}, T\right)$ in partial range; (c) $F_{2}\left(t_{d}, T\right)$ in full range; and (d) $F_{2}\left(t_{d}, T\right)$ in partial range.

For the feedback variable $i_{1}$ shown in Figure $6 \mathrm{a}, \mathrm{b}$, all of the deep-colored points in the curve represent the stable self-oscillating points, and the light-colored ones represent the unstable self-oscillating points. In Figure $6 \mathrm{~b}$, the upper intersection between the line $T=t_{d}$ and the stable part of the curve is the odd splitting point, and the lower is the even splitting point, the $U_{0}$ lags $i_{1}$ by exactly one period at these two points. So the splitting points can be reached by setting $t_{d}=T$ repeatedly, as in the iteration process shown by the red arrows. The system stabilizes at the odd splitting point with a longer initial delay, and at the even mode point with a shorter initial delay. Similarly, when using $i_{2}$ as the feedback variable in Figure $6 c, d$, the middle resonant point locates at the unique intersection between the line $T=4 t_{d}$ and the stable part of the curve. Thus, it can be reached by setting $t_{d}=T / 4$ repeatedly, which is equivalent to a $90^{\circ}$ phase shift in the feedback loop just like an integrator in Figure 4c. Only 2-3 iterations are needed if the initial delay is in the vicinity of the estimated target values, such as in Figure $6 \mathrm{~b}, \mathrm{~d}$, while more iterations are needed for a large deviated initial delay.

The resonant points can be found in a large frequency range even if the circuit parameters are unknown, so new resonant points can be reached when the parameters varies because the iteration is always in progress. Moreover, the error of the frequency measurement can be limited to the level of $0.002 \%$ easily (about $10 \mathrm{~Hz}$, for two decimals in $\mathrm{kHz}$ ), which is precise enough for the delay setting using controllable delay lines such as the DS1023-500. The precision of the frequency measurement is 
higher than that of the measurement of the voltage, current or reflected impedance amplitude, and the random error, due to the voltage fluctuation in the amplitude measurement, does not occur in the frequency measurement. This method can also be used on the occasion where the input voltage is unstable because no amplitude is measured, and the measurement and actuator are easy to realize, so the practicability is fairly high.

\section{Synchronous Tuning}

The purpose of circuit parameter tuning is to keep the resonant frequency of the TX equal to that of the $\mathrm{RX}$ all the time, i.e., $f_{\text {res }}=f_{\text {res1 }}=f_{\text {res2 }}$ :

$$
\left\{\begin{array}{l}
f_{\text {res } 1}=\frac{1}{2 \pi \sqrt{L_{1} C_{1}}} \\
f_{\text {res } 2}=\frac{1}{2 \pi \sqrt{L_{2} C_{2}}}
\end{array}\right.
$$

The frequency tuning here is automatic and affected by the circuit parameter variation during the circuit parameter tuning according to the analysis in Figure 5b. Thus, the function between the detuning and the phase difference of $i_{1}$ and $i_{2}$ is studied below, and an automatic circuit parameter tuning method with phase difference measurement and capacitance adjustment is proposed based on the function. The capacitor $C_{1}$ is chosen for adjustment because powering the actuator in the TX is easier than that in the RX.

Firstly, the frequency characteristic function for the automatic frequency tuning is obtained by setting the delay in the Hamel locus function $H_{d 2}(f)$ to $t_{d}=T / 4=1 / 4 f$. The capacitance $C_{1}$ is treated as an independent variable to derive the relation of $C_{1}$ and self-oscillating frequency $f$ as:

$$
F_{2}\left(C_{1}, f\right)=\left.H_{d 2}(f)\right|_{t_{d}=T / 4}=0
$$

and the function $f\left(C_{1}\right)$ is obtained by solving (12) and plotted in Figure 7. Substituting $s=j \omega$ in Equation (1) to obtain the Equation (13), and the $i_{1}$ and $i_{2}$ in the frequency domain can be obtained by solving Equation (13):

$$
\left\{\begin{array}{l}
\mathbf{I}_{1}\left(C_{1}, \omega\right)=\left.I_{1}(s)\right|_{s=j \omega} \\
\mathbf{I}_{2}\left(C_{1}, \omega\right)=\left.I_{2}(s)\right|_{s=j \omega}
\end{array}\right.
$$

where $C_{1}$ and $\omega$ are independent variables, and $\mathbf{I}_{\mathbf{1}}$ and $\mathbf{I}_{\mathbf{2}}$ are the current phasors.

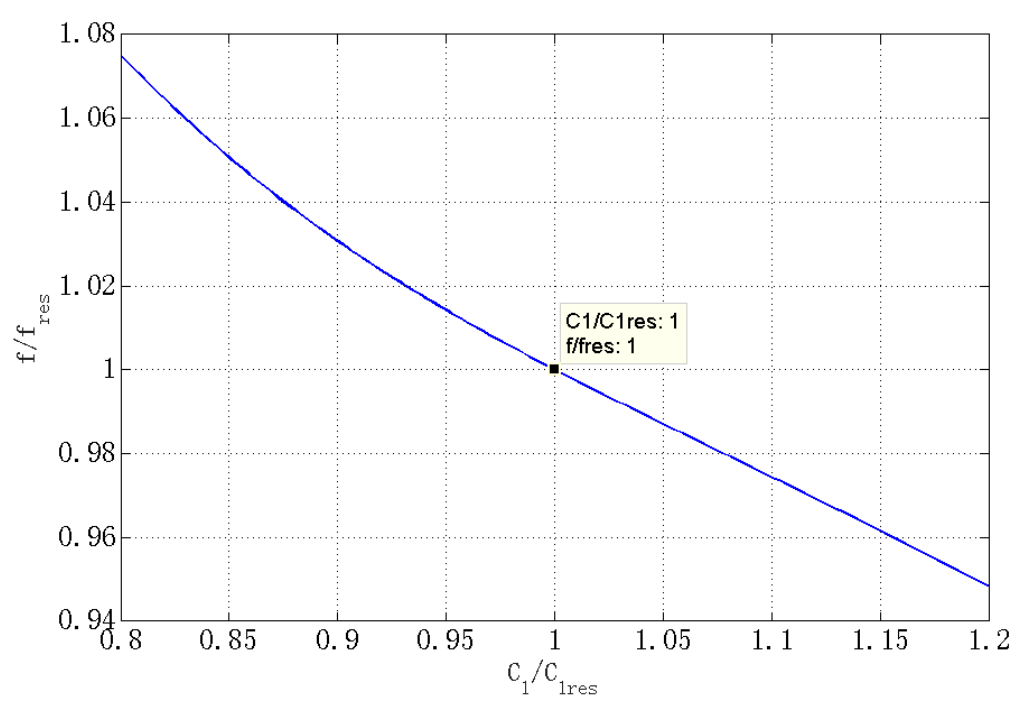

Figure 7. Function $f\left(C_{1}\right)$. 
Secondly, substituting the value of $C_{1}$ and its corresponding angular frequency $\omega=2 \pi f$ in the $f\left(C_{1}\right)$ in the $i_{1}\left(C_{1}, \omega\right)$ and $i_{2}\left(C_{1}, \omega\right)$ to obtain the functions of $C_{1}$ and the phase of $i_{1}$ and $i_{2}$, i.e., $\phi_{1}\left(C_{1}\right)$, and $\phi_{2}\left(C_{1}\right)$, and then the phase difference function $\Delta \phi\left(C_{1}\right)=\phi_{2}\left(C_{1}\right)-\phi_{1}\left(C_{1}\right)$, as shown in Figure 8.

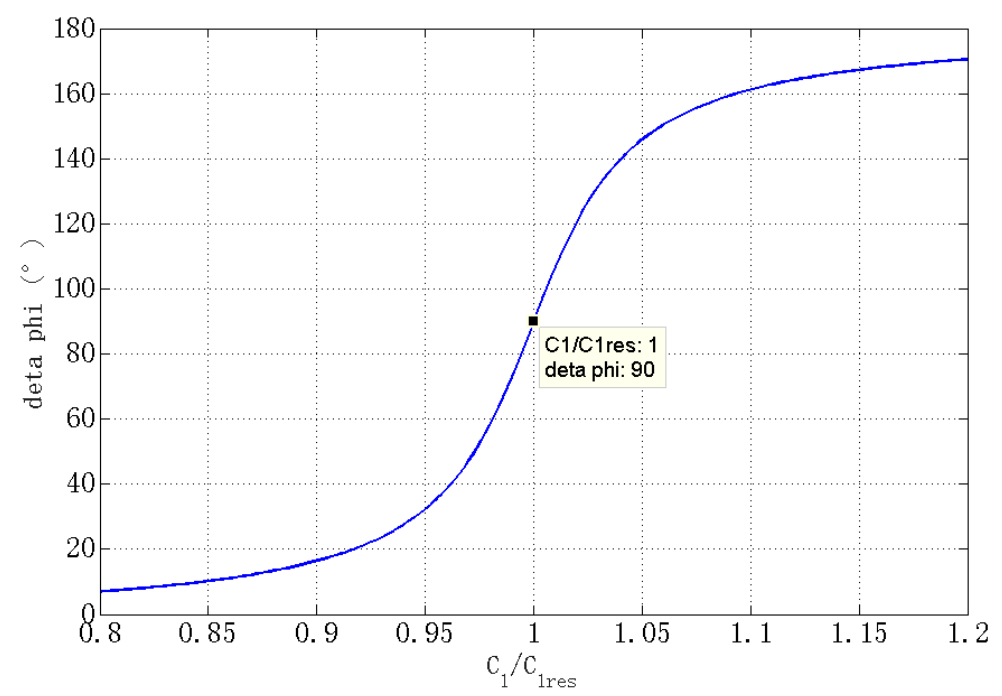

Figure 8. Function $\Delta \phi\left(C_{1}\right)$.

Above all, it is clear that this function is monotonic, and the circuit parameters are tuned only when $\Delta \phi=90^{\circ}$. Therefore, the circuit parameter tuning can be realized by the inspection of the $\Delta \phi$ and the adjustment of the $C_{1}$ to keep $\Delta \phi=90^{\circ}$ within the margin of error.

\section{Experimental Verification}

The system block diagram and experimental platform are shown in Figures 9 and 10, respectively. The synchronous tuning circuit includes the self-oscillating feedback loop, delay adjustment circuit, and capacitance adjustment circuit. The feedback loops for the feedback variable $i_{1}$ and $i_{2}$ include the current transformer $C T_{1}, C T_{2}$, zero-crossing comparator $Z C_{1}, Z C_{2}$, controllable delay line, and MOSFET driver. A wireless signal transfer module is needed to meet the demand of the transfer distance and rate in the feedback loop for $i_{2}$, such as the fast infrared module HSDL-3602 with the link distance $1.5 \mathrm{~m}$ and signal rate $4 \mathrm{Mb} / \mathrm{s}$, or radio frequency module [18] for further transfer distance and with a block between the TX and the RX. The delay adjustment includes the frequency measurement (FM) in the microcontroller unit (MCU) and the controllable delay line. The circuit parameter tuning circuit includes phase detector (PD) in the MCU and a variable capacitor adjusted by a stepper motor. Capacitor arrays or varicap diodes can also be used on different occasions [6,11,12,25]. The components and circuit parameters are listed in Tables 1 and 2, respectively. The high- $Q$ resonant tanks are comprised of copper pipes with large diameter and ceramic plate capacitors with low dielectric loss. The inductances and capacitances are measured by an inductance (L), capacitance (C) and resistance (R) meter (LCR meter) (TH2828, Tonghui Electronic Co. Ltd., Changzhou, Jiangsu, China), the equivalent resistances are measured by dividing the resonant voltage by the resonant current, and the middle resonant frequency, splitting frequency and the intrinsic delay are observed with an oscilloscope (TDS2024B, Tektronix Electronic Co. Ltd., Beaverton, OR, USA). The calculated values of these resonant frequencies are acquired using the measurement values of the components. The delay for the feedback variable $i_{1,2}$ includes the controllable delay $t_{d c}$ and the intrinsic delay $t_{d s 1,2}$, i.e., $t_{\mathrm{d}}=t_{d s 1,2}+t_{d c}$, and $t_{d s 1,2}=t_{d s \mathrm{~A} 1,2}+t_{d s \mathrm{~B}}$, marked in detail in Figure 9 . The required $t_{d}$ can be acquired by setting $t_{d c}=t_{d}-t_{d s 1,2}$ using the controllable delay line made of two DS1023-500 in cascade, with the delay step of $5 \mathrm{~ns}$. The delays of the $C T_{1,2}$ are far shorter than $5 \mathrm{~ns}$ and can be included within the $t_{d s 1,2}$ as a constant. 


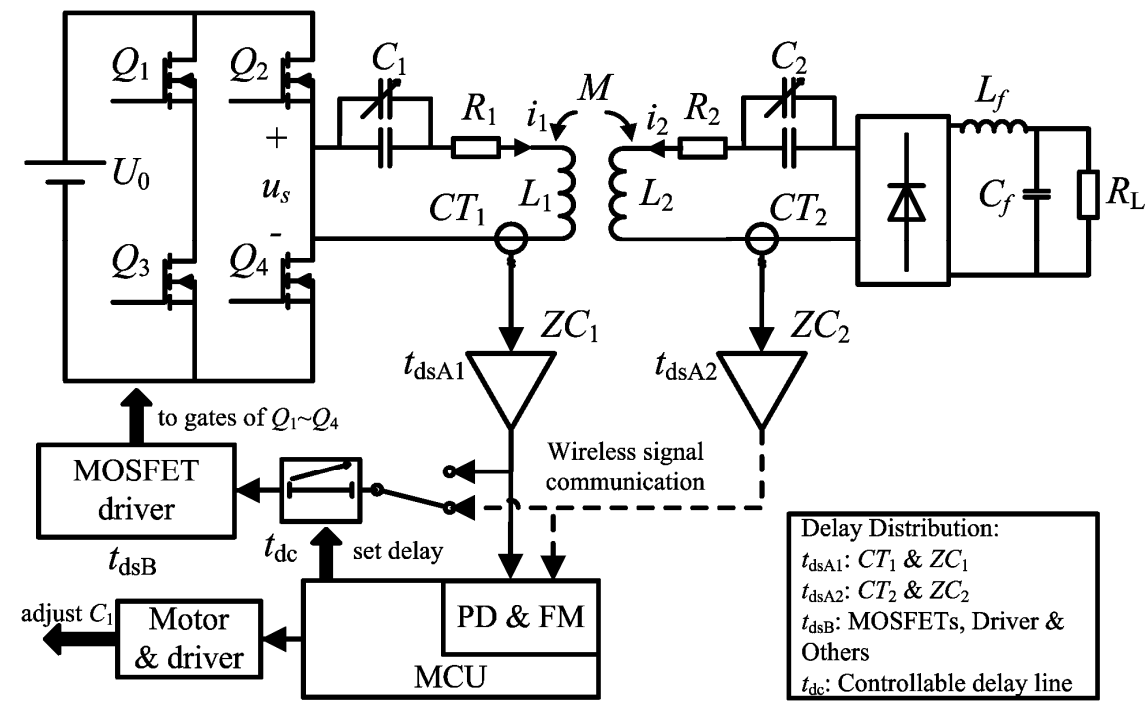

Figure 9. Block diagram of the experimental platform.

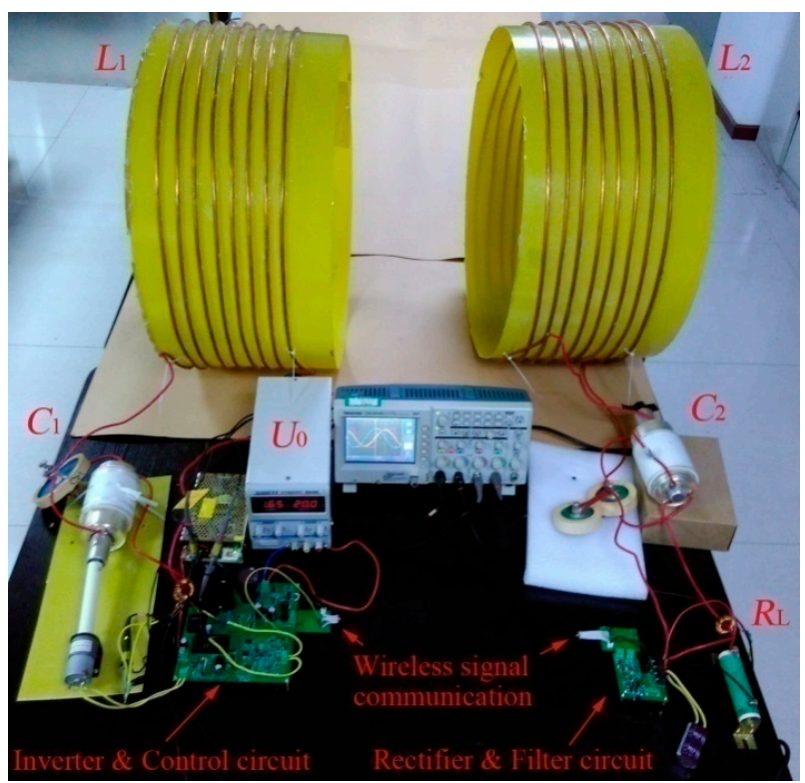

Figure 10. Photo of the experimental platform.

Table 1. Types of components.

\begin{tabular}{ccc}
\hline Part & Name & Material or Type \\
\hline \multirow{2}{*}{ Resonant tanks } & Coil & Copper pipe $\Phi=6 \mathrm{~mm}$ \\
& Fixed capacitor & Ceramic plate capacitor \\
& Variable capacitor & Vacuums adjustable capacitor \\
\hline \multirow{2}{*}{ Power circuit } & MOSFET & SCH2080KE \\
& MOSFET driver & IR2011 \\
& Rectifier diode & MBR3045 \\
\hline \multirow{2}{*}{ Feedback loop } & Delay line & DS1023-500 \\
& Comparator & MAX913 \\
& Infrared data communication & HSDL-3602 \\
\hline \multirow{2}{*}{ Control circuit } & MCU & STM32F405RE \\
& Motor driver & L298N \\
\hline
\end{tabular}


Table 2. Parameters of components.

\begin{tabular}{cccc}
\hline Symbol & Value & Symbol & Value \\
\hline$U_{0}(\mathrm{~V})$ & 20.0 & $k$ & 0.056 \\
$L_{1}, L_{2}(\mu \mathrm{H})$ & $49.1,49.5$ & $t_{\mathrm{ds} 1}, t_{\mathrm{ds} 2}(\mathrm{~ns})$ & 175,290 \\
$C_{1}, C_{2}(\mathrm{pF})$ & $1774.4,1759.6$ & $f_{\text {res }}, f_{\text {odd }}, f_{\text {even }}(\mathrm{kHz})($ measurement $)$ & $539.69,530.79,547.98$ \\
$R_{1}, R_{2}, R_{\mathrm{L}}(\Omega)$ & $0.73,0.54,10.32$ & $f_{\text {res }}, f_{\text {odd }}, f_{\text {even }}(\mathrm{kHz})($ calculation $)$ & $539.20,532.63,546.75$ \\
\hline
\end{tabular}

The flow chart of the automatic delay compensation is shown in Figure 11 according to the analysis in Section 3. The estimated value of the initial delay $t_{\mathrm{d}}(0)$ is set to the vicinity of $T_{\text {res }}$ for the feedback variable $i_{1}$, and to the vicinity of $T_{\text {res }} / 4$ for the feedback variable $i_{2}$ in order to achieve a short stabilization time. The iteration process is shown in Figure 12, and the range of delay, period, and frequency are measured and listed in Table 3. It can be seen that for the feedback variable $i_{1}$, the system stabilizes at the odd splitting point under a long initial delay, and at the even splitting point under a short initial delay; and for $i_{2}$, the system stabilizes at the middle resonant point regardless of the initial delay. The tuning process is not only fully automatic with the repetitive loop, just as is shown in Figure 1b, but also fast because only 2-3 iterations are needed for $i_{1}$, and 1-2 iterations for $i_{2}$.

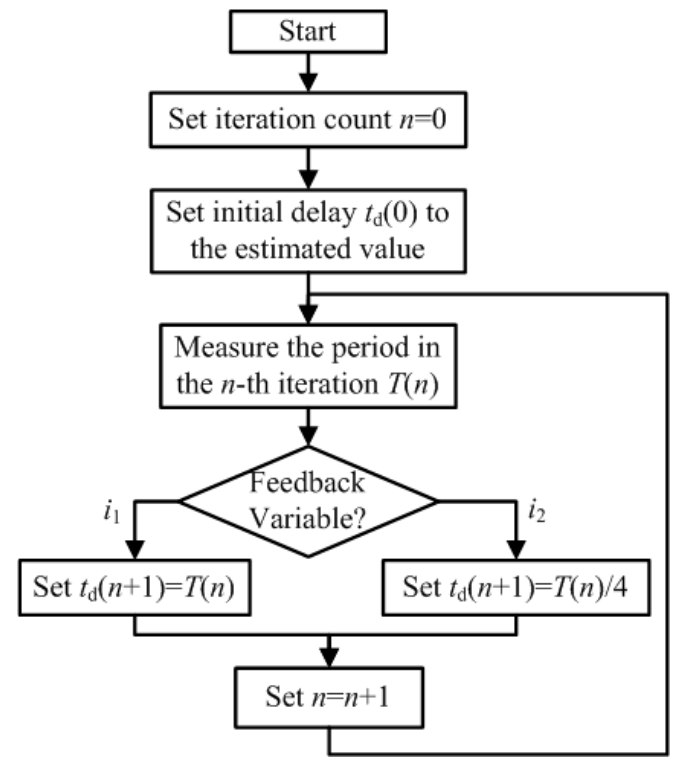

Figure 11. Flow chart of the automatic delay compensation.

The Figure 13 shows the variation tendency of the self-oscillating frequency with $C_{1}$ or $C_{2}$, for the feedback variable $i_{1}$ or $i_{2}$, respectively. In Figure 13a, for the feedback variable $i_{1}$, the system is initially tuned in the odd splitting point. When $C_{1}$ increases from the tuned value $C_{1 \text { res }}$ to a certain amount, the frequency will hop to the vicinity of the even mode, and the frequency is tuned to the even mode when $C_{1}$ decreases to $C_{1 \text { res }}$ again, as shown in the track ABCDE. The track EFGHA shows the frequency hopping from the even to odd splitting point. The similar frequency variation process with the change of $C_{2}$ is shown in Figure 13b, while in Figure $13 \mathrm{c}, \mathrm{d}$, for the feedback variable $i_{2}$, the system always goes back to the middle resonant point after the $C_{1}$ or $C_{2}$ is retuned, so the robustness for $i_{2}$ is stronger. The variation trends of the self-oscillating frequency in the experiments are in good agreement with the analysis in Section 2, and the transfer power and efficiency shown in Table 4 indicate that though the power is lower, the efficiency is higher at the middle resonant point. 


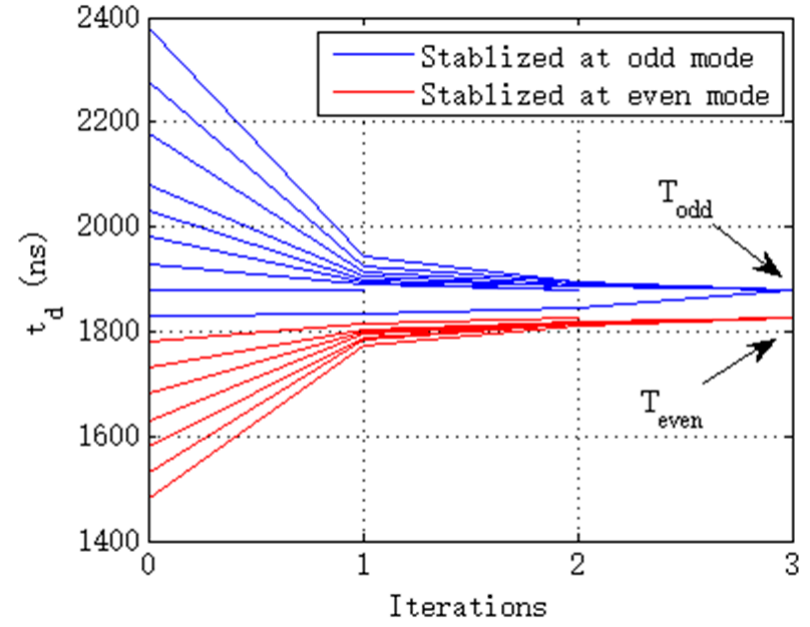

(a)

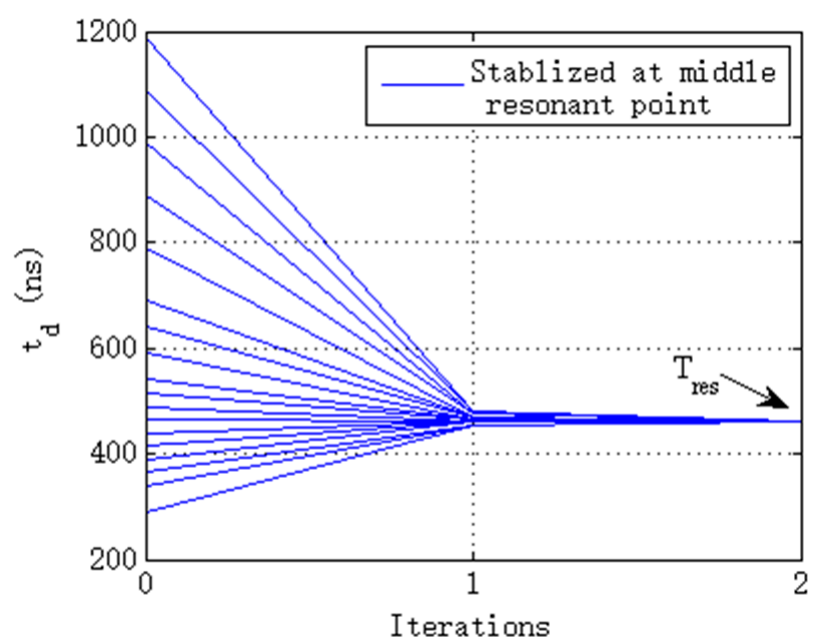

(b)

Figure 12. The iteration process of $t_{\mathrm{d}}$ with different feedback variables, at different initial delay: (a) feedback variable $i_{1}$; and (b) feedback variable $i_{2}$.

Table 3. Range of delay, period, and frequency in each step of the delay compensation.

\begin{tabular}{|c|c|c|c|c|c|c|c|c|c|}
\hline \multirow{2}{*}{ Iteration Count $n$} & \multicolumn{3}{|c|}{ Feedback Variable $i_{1}$ (Odd Splitting Frequency) } & \multicolumn{3}{|c|}{ Feedback Variable $i_{1}$ (Even Splitting Frequency) } & \multicolumn{3}{|c|}{ Feedback Variable $i_{2}$} \\
\hline & $t_{\mathrm{d}}(\mathrm{ns})$ & $T$ (ns) & $f(\mathbf{k H z})$ & $t_{\mathrm{d}}(\mathrm{ns})$ & $T$ (ns) & $f(\mathbf{k H z})$ & $t_{\mathrm{d}}(\mathrm{ns})$ & $T(\mathrm{~ns})$ & $f(\mathbf{k H z})$ \\
\hline 0 & $1830-2380$ & $1837.06-1947.55$ & $544.34-513.46$ & $1480-1780$ & 1778.13-1813.32 & $562.39-551.48$ & $290-1190$ & 1823.96-1921.01 & $548.26-520.56$ \\
\hline 1 & 1835-1945 & $1847.47-1897.11$ & $541.28-527.11$ & $1775-1815$ & $1811.53-1823.81$ & $552.01-548.30$ & $455-480$ & $1850.85-1849.68$ & $540.29-540.63$ \\
\hline 2 & 1845-1895 & $1877.66-1882.28$ & $532.57-531.27$ & $1810-1825$ & $1823.65-1824.88$ & $548.35-547.98$ & 460 & 1852.92 & 539.69 \\
\hline 3 & 1880 & 1883.98 & 530.79 & 1825 & 1824.88 & 547.98 & & & \\
\hline
\end{tabular}




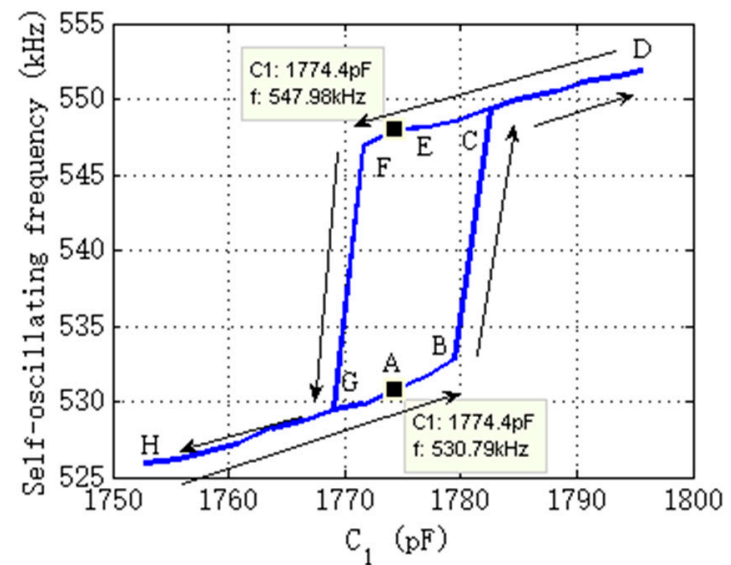

(a)

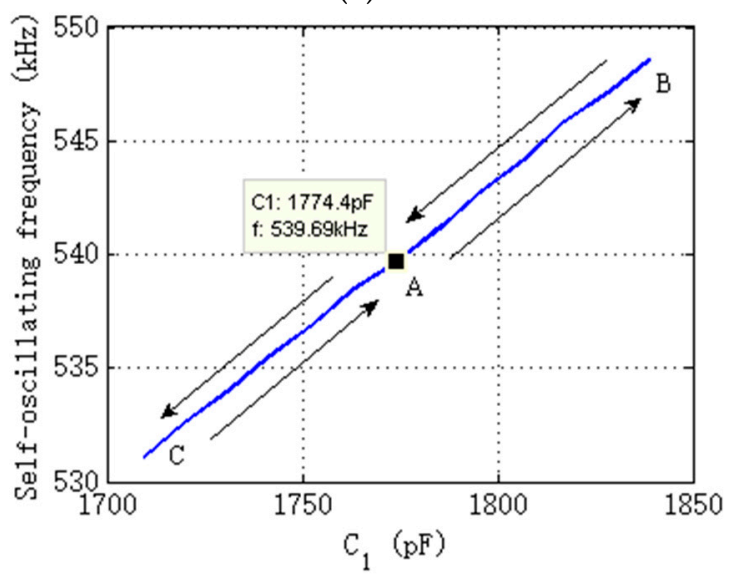

(c)

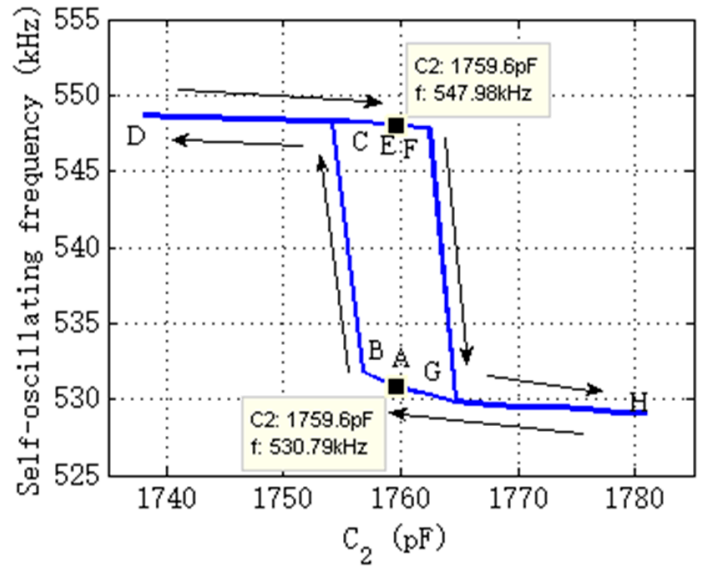

(b)

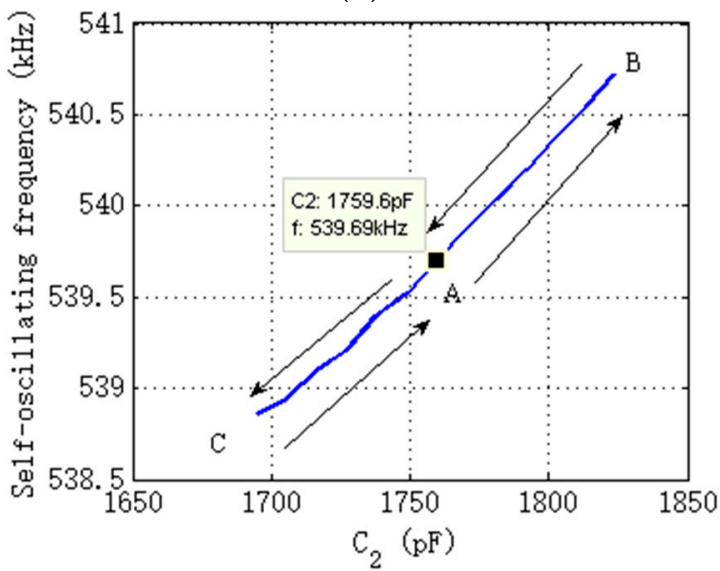

(d)

Figure 13. Variation trends of self-oscillating frequency in different feedback mode. (a) Feedback variable $i_{1}$, adjust $C_{1} ;(\mathbf{b})$ feedback variable $i_{1}$, adjust $C_{2} ;(\mathbf{c})$ feedback variable $i_{2}$, adjust $C_{1}$; and (d) feedback variable $i_{2}$, adjust $C_{2}$.

Table 4. Measurement results of power and efficiency.

\begin{tabular}{cccc}
\hline Feedback Variable & $\boldsymbol{i}_{\mathbf{1}}$ (Odd Splitting Point) & $\boldsymbol{i}_{\mathbf{1}}$ (Even Splitting Point) & $\boldsymbol{i}_{\mathbf{2}}$ \\
\hline Power $(\mathrm{W})$ & 37.0 & 36.8 & 30.2 \\
Efficiency $(\%)$ & 83.5 & 83.8 & 87.2 \\
\hline
\end{tabular}

The waveforms of $u_{\mathrm{s}}, i_{1}$, and $i_{2}$ for the automatic circuit parameter tuning are shown in Figure 14 . The current values are obtained by measuring the voltage across the sampling resistor in the current transformer and displayed as the voltage value in the oscilloscope. In Figure $14 \mathrm{a}, C_{2}$ is set to $1823.6 \mathrm{pF}$, which is $64.0 \mathrm{pF}$ larger than that of the original resonant value to represent the circuit parameter tuning, and the phase different of $i_{1}$ and $i_{2}$ is far less than $90^{\circ}$. Thus, the new resonant value of $C_{1}$ needs to be increased according to the analysis in Figure 8. In Figure $14 \mathrm{~b}-\mathrm{d}$, the phase difference approaches to $90^{\circ}$ gradually, and finally comes into the range of $90^{\circ} \pm 1^{\circ}$. The frequency detuning exists for a short time, but disappears after the automatic circuit parameter tuning. The amplitude of $i_{2}$ almost remains constant in the process, indicating that the received power is stable in a certain range of detuning during the tuning process. 


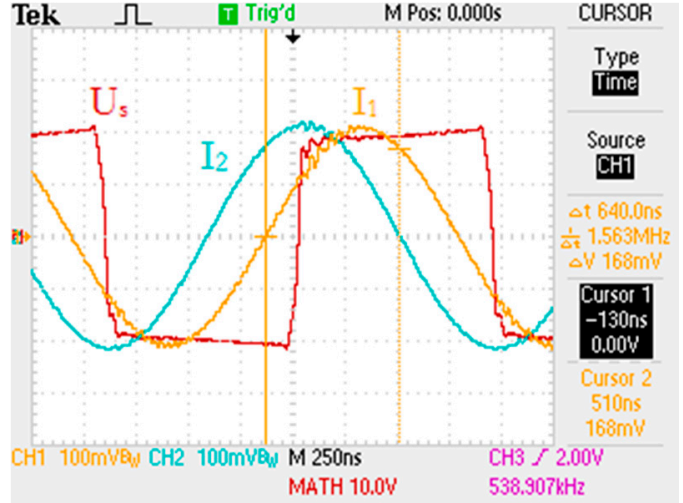

(a)

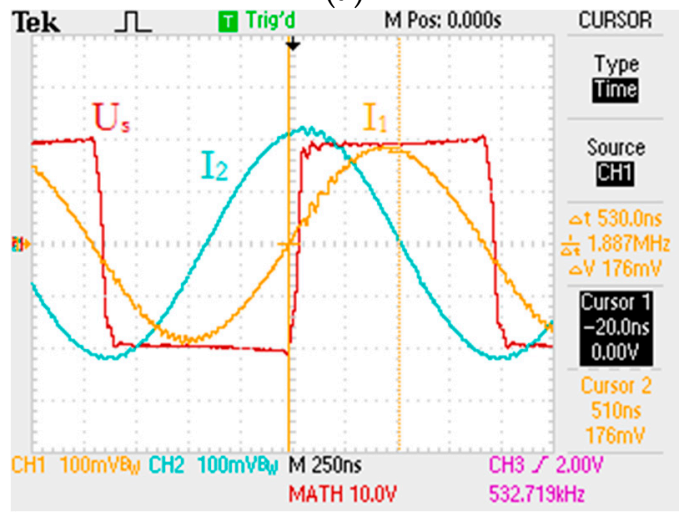

(c)

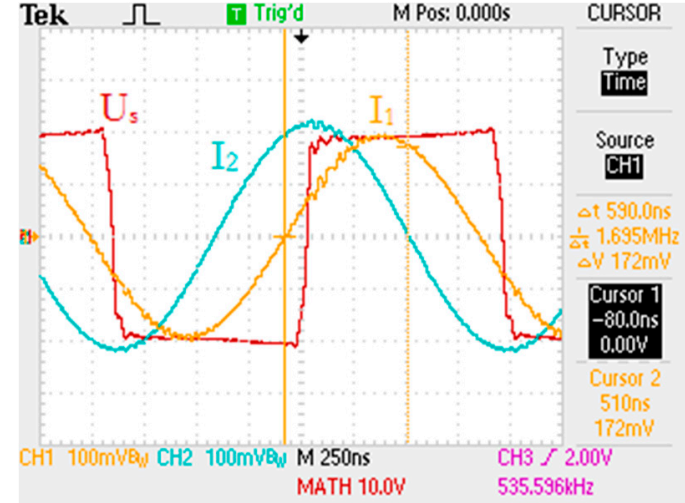

(b)

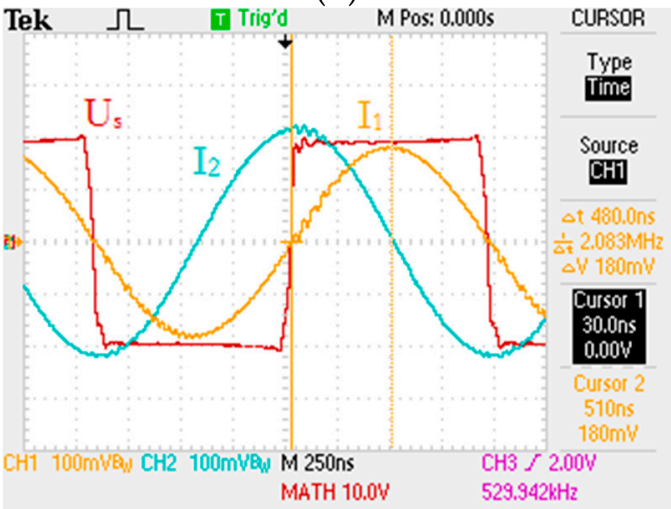

(d)

Figure 14. Waveforms of the voltage $u_{\mathrm{s}}$ of the inverter, and the currents $i_{1}$ and $i_{2}$ in the TX and $\mathrm{RX}$ during the circuit parameter tuning process. (a) $C_{1}=1774.4 \mathrm{pF}, \Delta \phi=56.4^{\circ}$; (b) $C_{1}=1794.9 \mathrm{pF}$, $\Delta \phi=65.9^{\circ} ;$ (c) $C_{1}=1816.5 \mathrm{pF}, \Delta \phi=80.2^{\circ} ;$ and $(\mathbf{d}) C_{1}=1838.7 \mathrm{pF}, \Delta \phi=89.4^{\circ}$.

\section{Conclusions}

The self-oscillating characteristics of a close-coupling WPT system for the feedback variables TX current and RX current are analyzed. It is proved by both theory and experiments that the system operates more stably and efficiently at the middle resonant point for the feedback variable RX current than at the odd or even splitting point for the feedback variable TX current, and the frequency-hopping caused by the frequency-splitting is eliminated. The detuning due to the intrinsic delay are solved by the automatic delay compensation with the iteration of delay and period, and the detuning due to the circuit parameter variation are solved by the automatic circuit parameter tuning with the function of detuning and phase difference. The frequency and circuit parameter tuning can be used at the same time to realize the synchronous tuning. The tuning is fully automatic and precise, and is suitable for practical applications where circuit parameter values are easy to change.

The proposed frequency and circuit parameter tuning can be used not only in WPT systems, but also in other applications using high frequency resonant inverters, such as inductive heating or electronic ballast. Using the Hamel locus to analyze the frequency characteristic and the circuit parameter influence can also be popularized to the related areas.

Acknowledgments: The research was supported by the Experimental teaching and research center in the School of Electrical Engineering, Wuhan University.

Author Contributions: Po Hu modeled the system and revised the paper; Jieshuai Ren researched the synchronous tuning method and wrote the initial draft; Jieshuai Ren and Wenan Li established the experimental platform, conducted the experiments and analyzed the experimental data.

Conflicts of Interest: The authors declare no conflict of interest. 


\section{Abbreviations}

The following abbreviations are used in this manuscript:

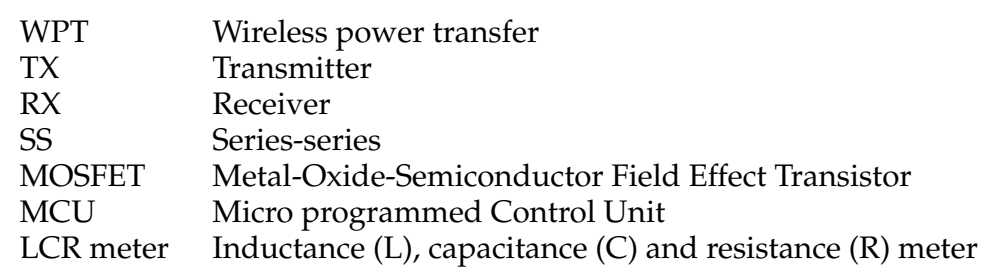

\section{References}

1. Sample, A.P.; Meyer, D.A.; Smith, J.R. Analysis, Experimental Results, and Range Adaptation of Magnetically Coupled Resonators for Wireless Power Transfer. IEEE Trans. Ind. Electron. 2011, 58, 544-554. [CrossRef]

2. Brusamarello, V.J.; Blauth, Y.B.; Azambuja, R.; Mueller, I. A Study on Inductive Power Transfer with Wireless Tuning. In Proceedings of the IEEE Instrumentation and Measurement Technology Conference, Graz, Austria, 13-16 May 2012; pp. 1098-1103.

3. Li, Q.; Liang, Y.C. An Inductive Power Transfer System with a High-Q Resonant Tank for Mobile Device Charging. IEEE Trans. Power Electron. 2015, 30, 6203-6212. [CrossRef]

4. Moorey, C.; Holderbaum, W.; Potter, B. Investigation of High-Efficiency Wireless Power Transfer Criteria of Resonantly-Coupled Loops and Dipoles through Analysis of the Figure of Merit. Energies 2015, 8, 11342-11362. [CrossRef]

5. Kurs, A.; Karalis, A.; Moffatt, R.; Joannopoulos, J.D.; Fisher, P.; Soljacic, M. Wireless Power Transfer via Strongly Coupled Magnetic Resonances. Science 2007, 317, 83-86. [CrossRef] [PubMed]

6. Thuc, P.D.; Lee, J. A Dynamically Adaptable Impedance-Matching System for Midrange Wireless Power Transfer with Misalignment. Energies 2015, 8, 7593-7617.

7. Zhang, Y.; Lu, T.; Zhao, Z.; He, F.; Chen, K.; Yuan, L. Employing Load Coils for Multiple Loads of Resonant Wireless Power Transfer. IEEE Trans. Power Electron. 2015, 30, 6174-6181. [CrossRef]

8. Johari, R.; Krogmeier, J.V.; Love, D.J. Analysis and Practical Considerations in Implementing Multiple Transmitters for Wireless Power Transfer via Coupled Magnetic Resonance. IEEE Trans. Ind. Electron. 2014, 61, 1774-1783. [CrossRef]

9. Dai, X.; Sun, Y. An Accurate Frequency Tracking Method Based on Short Current Detection for Inductive Power Transfer System. IEEE Trans. Ind. Electron. 2014, 61, 776-783. [CrossRef]

10. Wang, J.; Zhu, Z.; Li, C.; Huangfu, J.; Ran, L. PLL-Based Self-Adaptive Resonance Tuning for a Wireless-Powered Potentiometer. IEEE Trans. Circuits Syst. II 2013, 60, 392-396. [CrossRef]

11. Kim, J.; Jeong, J. Range-Adaptive Wireless Power Transfer Using Multiloop and Tunable Matching Techniques. IEEE Trans. Ind. Electron. 2015, 62, 6233-6241. [CrossRef]

12. Bac, X.N.; Vilathgamuwa, D.M.; Foo, G.H.B.; Wang, P.; Ong, A.; Madawala, U.K.; Trong, D.N. An Efficiency Optimization Scheme for Bidirectional Inductive Power Transfer Systems. IEEE Trans. Power Electron. 2015, 30, 6310-6319.

13. Wang, B.; Hu, A.P.; Budgett, D. Maintaining middle zero voltage switching operation of parallel-parallel tuned wireless power transfer system under bifurcation. IET Power Electron. 2014, 7, 78-84. [CrossRef]

14. Gao, Y.; Farley, K.; Tse, Z. A Uniform Voltage Gain Control for Alignment Robustness in Wireless EV Charging. Energies 2015, 8, 8355-8370. [CrossRef]

15. Teck, C.B.; Kato, M.; Imura, T.; Sehoon, O.; Hori, Y. Automated Impedance Matching System for Robust Wireless Power Transfer via Magnetic Resonance Coupling. IEEE Trans. Ind. Electron. 2013, 60, 3689-3698.

16. Ziaie, B.; Rose, S.C.; Nardin, M.D.; Najafi, K. A Self-Oscillating Detuning-Insensitive Class-E Transmitter for Implantable Microsystems. IEEE Trans. Biomed. Eng. 2001, 48, 397-400. [CrossRef] [PubMed]

17. Tang, C.; Sun, Y.; Dai, X.; Wang, Z.; Su, Y.; Hu, A. Transition control of bifurcated frequencies in inductive power transfer systems through time delay perturbation. Acta Phys. Sin.-Chin. Ed. 2013, 62, 508-517. (In Chinese)

18. Li, H.; Li, J.; Wang, K.; Chen, W.; Yang, X. A Maximum Efficiency Point Tracking Control Scheme for Wireless Power Transfer Systems Using Magnetic Resonant Coupling. IEEE Trans. Power Electron. 2015, 30, 3998-4008. [CrossRef] 
19. Zhang, Y.; Zhao, Z. Frequency Splitting Analysis of Two-Coil Resonant Wireless Power Transfer. IEEE Antennas Wirel. Propag. Lett. 2014, 13, 400-402. [CrossRef]

20. Huang, R.; Zhang, B.; Qiu, D.; Zhang, Y. Frequency Splitting Phenomena of Magnetic Resonant Coupling Wireless Power Transfer. IEEE Trans. Magn. 2014, 50, 1-4. [CrossRef]

21. Williams, D.; Bingham, C.; Foster, M.; Stone, D. Hamel locus design of self-oscillating DC-DC resonant converters. IET Power Electron. 2010, 3, 86-94. [CrossRef]

22. Yan, K.; Chen, Q.; Hou, J.; Ren, X.; Ruan, X. Self-Oscillating Contactless Resonant Converter with Phase Detection Contactless Current Transformer. IEEE Trans. Power Electron. 2014, 29, 4438-4449. [CrossRef]

23. Ren, J.; Hu, P.; Yang, D.; Liu, D. Tuning of Mid-Range Wireless Power Transfer System Based on Delay-Iteration Method. IET Power Electron. 2016, 9, 1563-1570. [CrossRef]

24. Gille, J.C.; Pelegrin, M.J.; Decaulne, P. Feedback Control Systems: Analysis, Synthesis, and Design; McGraw-Hill: New York, NY, USA, 1959; pp. 446-462.

25. Colak, K.; Asa, E.; Bojarski, M.; Czarkowski, D.; Onar, O.C. A Novel Phase-Shift Control of Semibridgeless Active Rectifier for Wireless Power Transfer. IEEE Trans. Power Electron. 2015, 30, 6288-6297. [CrossRef]

26. Niu, W.; Chu, J.; Gu, W.; Shen, A. Exact Analysis of Frequency Splitting Phenomena of Contactless Power Transfer Systems. IEEE Trans. Circuits Syst. I Regul. Pap. 2013, 60, 1670-1677. [CrossRef]

(C) 2016 by the authors; licensee MDPI, Basel, Switzerland. This article is an open access article distributed under the terms and conditions of the Creative Commons Attribution (CC-BY) license (http://creativecommons.org/licenses/by/4.0/). 